\title{
Guest editorial for the topical collection: sustainable development and utilization of geothermal systems
}

\author{
P. G. Ranjith - D. Chandrasekharam - Ilmutdin Abdulagatov • Bisheng Wu • \\ Gnamani Pabasara Kumari Wanniarachchige
}

Published online: 4 November 2020

(C) Springer Nature Switzerland AG 2020

\section{Introduction}

In recent years, climate change has accelerated progressive renewable energy programs, including the Sustainable Energy for All Initiative (UN), the United States Environmental Protection Agency's Green Power Partnership, and the European Green Deal, the main aims of which are to reduce anthropogenic greenhouse gas emissions and dependence on fossil fuels and enhance resource efficiency. Geothermal energy, which utilizes the thermal energy stored in the earth's crust, can play a significant role in the transition from fossil fuels as it is clean energy which is continuously renewed. The heat stored in the earth's crust within a $10 \mathrm{~km}$ depth is estimated to contain

P. G. Ranjith $(\bowtie) \cdot$ G. P. K. Wanniarachchige

Deep Earth Energy Laboratory, Civil Engineering Dept,

Monash University, Clayton, Australia

e-mail: ranjith.pg@monash.edu

D. Chandrasekharam

Department of Earth Sciences, Indian Institute of

Technology Hyderabad, Kandi, India

I. Abdulagatov

Thermophysical Division at the Geothermal Research Institute of the Russian Academy of Sciences, Dagestan State University, Makhachkala, Russia

B. $\mathrm{Wu}$

State Key Laboratory of Hydroscience and Engineering, Department of Hydraulic Engineering, Tsinghua

University, Beijing 100084, China
50,000 times more energy than all the worldwide energy from oil and other gas resources (Shere 2013). Of the other renewables, geothermal energy also plays an important role by being available in all seasons of the year, irrespective of the weather conditions, whereas solar and wind energy exhibit great variability. In terms of total $\mathrm{CO}_{2}$ emissions during the lifecycle of a geothermal power plant and the equivalent solar PV, geothermal energy will also have a substantial net positive impact on the environment (Chandrasekharam and Pathegama 2020).

Heat, permeability, and fluids are the key elements of a commercially-viable geothermal resource. The geothermal resources with the greatest potential lie close to tectonic boundaries, volcanoes, and locations where the crust is fractured, causing heat sources to be accessible (DiPippo 2012). In these systems, significant volumes of fluid are available and the heat of the earth is carried to the surface by convective circulation, the energy coming in either vapor-dominant or liquid-dominant forms. The utilization of deep/unconventional geothermal resources is currently a great challenge for the geothermal industry, and it requires high levels of innovation, research, and development and technological advances. Critical challenges which demand basic and applied research, technological development, and demonstration include enhancing the permeability of rock by different stimulation technologies (hydraulic, thermal, and chemical) to enlarge the extent of productive geothermal fields, improving reservoir exploration and drilling 
technology for deep geothermal reservoirs, improving reservoir assessment, analysis, and modelling of the coupled thermo-hydro-chemical-mechanical processes in geothermal reservoirs, and introducing innovative fracturing and circulation fluids, including supercritical fluid systems (Isaka et al. 2019; Kumari and Ranjith 2019; Tester et al. 2006).

The production of commercially-viable geothermal power is the main challenge faced by current conventional/unconventional development due to the lack of understanding of the governing factors and their influence on each phase of projects. Decision-makers, together with the general public, demand assurance that the technical and economical challenges and the environmental risks associated with resource exploration, deep drilling, stimulation, seismic control, and long-term production (which involve multiphase flow, coupled with chemical, thermal, and mechanical interactions between fluids and reservoir rocks) are fully researched.

\section{Scope of the special issue/topical collection}

This special issue aims to address these challenges. We have collected papers on recent developments in resource exploration and assessment, detailed modelling, and state-of-the-art experimental approaches, which together will guide the design and implementation of future geothermal developments. Devoted to sustainable development and the utilization of geothermal systems, the issue includes contributions that advance our understanding of the modeling of hydraulic fracture initiation and propagation, coupled thermal-mechanical-fluid flow systems, the use of innovative fracturing and circulation fluids, the innovative conceptualization of future geothermal systems, and balanced overviews of the field. The special issue has been very successful in publishing 17 excellent papers covering both fundamental and applied state-of-the-art works in the geothermal discipline. Some papers (e.g. $\mathrm{CO}_{2}$ emissions from renewables: solar PV, hydrothermal and EGS sources) have already have received significant media coverage. The Guest Editors are very much appreciating and acknowledging the contributions from the authors and their support during the peer-review stages. Also, special thanks go to the Springer team of the G4 journal, for their support in the publication process of the special issue contributions.

We outline the contributions below.

1. "Deep geothermal resource assessment of the St. Lawrence Lowlands sedimentary basin (Québec) based on 3D regional geological modelling" by Bédard et al. (2020).

2. "Numerical investigation of the effects of the fracture network pattern on the heat extraction capacity of dual horizontal wells in enhanced geothermal systems" by Xin et al. (2020).

3. "Energy potential of a single-fracture, robust, engineered geothermal system" by Danko et al. (2020).

4. "Geothermal energy potential of Indian oilfields" by Singh (2020).

5. "Conceptualization and evaluation of the exploration and utilization of low/medium-temperature geothermal energy: a case study of the Guangdong-Hong Kong-Macao Greater Bay Area" by Xie et al. (2020).

6. "Potential of $\mathrm{CO}_{2}$-based geothermal energy extraction from hot sedimentary and dry rock reservoirs, and enabling carbon geo-sequestration" by Singh et al. (2020b).

7. " $\mathrm{CO}_{2}$ emissions from renewables: solar $\mathrm{PV}$, hydrothermal and EGS sources" by Chandrasekharam and Pathegama (2020).

8. "Near-room-temperature thermoelectric materials and their application prospects in geothermal power generation" by Yu et al. (2020).

9. "Studies of the heat and mass transfer phenomena when flowing a vapor-water mixture through the system of a geothermal reservoirwell" by Alkhasov et al. (2020b).

10. "A preliminary investigation of the assessment of geothermal potential at Eastern Peninsular India" by Singh et al. (2020a).

11. "Temperature effect on thermal-diffusivity and heat-capacity and derived values of thermalconductivity of reservoir rock materials" by Abdulagatova et al. (2020).

12. "Technologies of geothermal resources development in south Russia” by Alkhasov et al. (2020a).

13. "Excavation-based enhanced geothermal system (EGS-E): introduction to a new concept" by Zhao et al. (2020). 
14. "Technological design and efficiency assessment of heat production from the dry rock with different energy potential" by Alkhasova (2020).

15. “A global review of deep geothermal energy exploration: from a view of rock mechanics and engineering" by Zhang and Zhao (2020).

16. "Use of abandoned oil wells in geothermal systems in Turkey" by Kaplanoğlu et al. (2020).

17. "An analytical model for heat extraction through multi-link fractures of an enhanced geothermal system" by Tang et al. (2020).

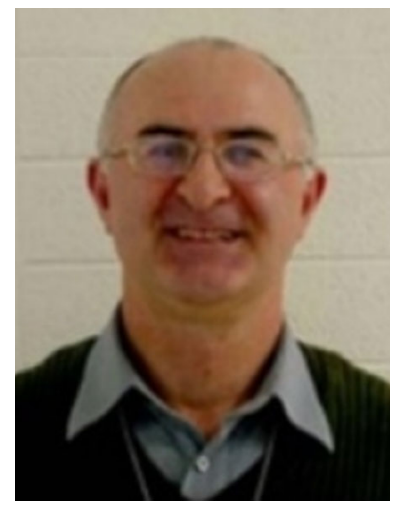

Prof. Ilmutdin Abdulagatov

\section{Guest Editorial Team}

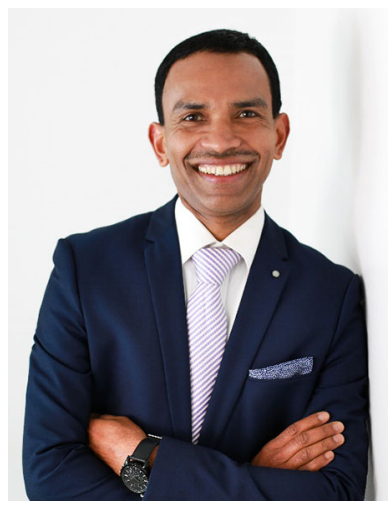

Prof. Ranjith Pathegama Gamage

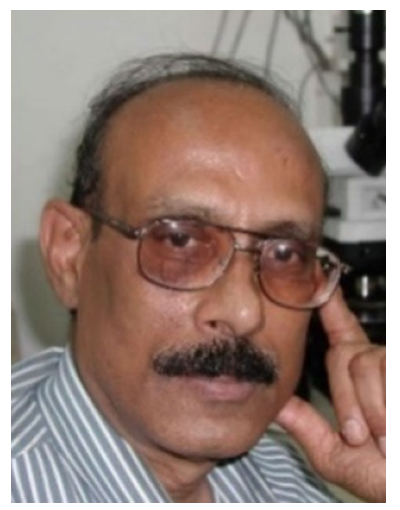

Prof. D. Chandrasekharam

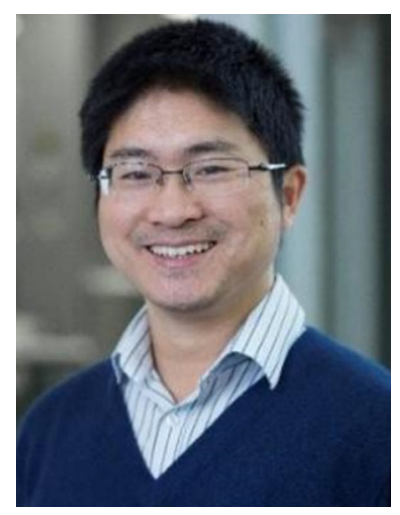

Assoc. Prof. Bisheng Wu

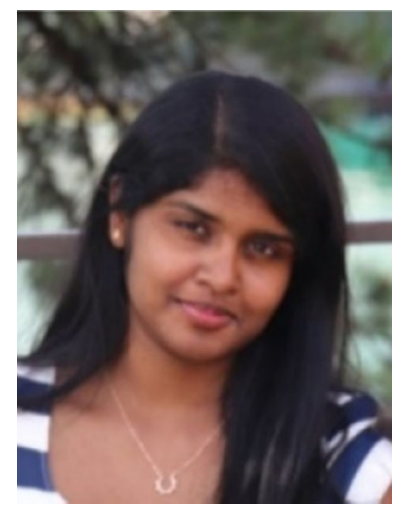

Dr. Pabasara Wanniarachchige 


\section{References}

Abdulagatova ZZ, Kallaev SN, Omarov ZM, Bakmaev AG, Grigor'ev BA, Abdulagatov IM (2020) Temperature effect on thermal-diffusivity and heat-capacity and derived values of thermal-conductivity of reservoir rock materials. Geomech Geophys Geo-energ Geo-resour 6(1):8

Alkhasov AB, Alkhasova DA, Ramazanov A (2020a) Technologies of geothermal resources development in the South of Russia. Geomech Geophys Geo-energ Geo-resour 6(1):7

Alkhasov A, Bulgakova N, Ramazanov M (2020b) Studies of the heat and mass transfer phenomena when flowing a vapor-water mixture through the system of the geothermal reservoir-well. Geomech Geophys Geo-energ Geo-resour 6(1): 11

Alkhasova DA (2020) Technological design and efficiency assessment of heat production from dry rock with different energy potential. Geomech Geophys Geo-energ Geo-resour 6(1):5

Bédard K, Comeau FA, Raymond J, Gloaguen E, Malo M, Richard MA (2020) Deep geothermal resource assessment of the St. Lawrence Lowlands sedimentary basin (Québec) based on 3D regional geological modelling. Geomech Geophys Geo-energ Geo-resour 6(3):1-18

Chandrasekharam D, Pathegama GR (2020) $\mathrm{CO}_{2}$ emissions from renewables: solar pv, hydrothermal and EGS sources. Geomech Geophys Geo-energ Geo-resour 6(1):13

Danko G, Jobbik A, Baracza MK, Varga G, Kovacs I, Wittig V (2020) Energy potential of a single-fracture, robust, engineered geothermal system. Geomech Geophys Geo-energ Geo-resour 6(1):1-14

DiPippo R (2012) Geothermal power plants: principles, applications, case studies and environmental impact. Butterworth-Heinemann, Oxford

Isaka BA, Ranjith PG, Rathnaweera TD (2019) The use of super-critical carbon dioxide as the working fluid in enhanced geothermal systems (EGSs): a review study. Sustain Energy Technol Assess 36:100547

Kaplanoğlu MA, Baba A, Akkurt GG (2020) Use of abandoned oil wells in geothermal systems in Turkey. Geomech Geophys Geo-energ Geo-resour 6(1):2

Kumari WGP, Ranjith PG (2019) Sustainable development of enhanced geothermal systems based on geotechnical research-A review. Earth Sci Rev 199:102955

Shere J (2013) Renewable: the world-changing power of alternative energy. Macmillan, New York
Singh HK (2020) Geothermal energy potential of Indian oilfields. Geomech Geophys Geo-energ Geo-resour 6(1):19

Singh HK, Sinha SK, Chandrasekharam D (2020a) A preliminary investigation for the assessment of geothermal potential at Eastern Peninsular India. Geomech Geophys Geo-energ Geo-resour 6(1):9

Singh M, Tangirala SK, Chaudhuri A (2020b) Potential of CO2 based geothermal energy extraction from hot sedimentary and dry rock reservoirs, and enabling carbon geo-sequestration. Geomech Geophys Geo-energ Geo-resour 6(1):16

Tang Y, Ma T, Chen P, Ranjith PG (2020) An analytical model for heat extraction through multi-link fractures of the enhanced geothermal system. Geomech Geophys Geo-energ Geo-resour 6(1): 1

Tester JW, Anderson BJ, Batchelor AS, Blackwell DD, DiPippo R, Drake EM (2006) The future of geothermal energy: Impact of enhanced geothermal systems (EGS) on the United States in the 21st century. Massachusetts Institute of Technology, Cambridge

Xie H, Li C, Zhou T, Chen J, Liao J, Ma J, Li B (2020) Conceptualization and evaluation of the exploration and utilization of low/medium-temperature geothermal energy: a case study of the Guangdong-Hong Kong-Macao Greater Bay Area. Geomech Geophys Geo-energ Geo-resour 6(1):18

Xin Y, Zhuang L, Sun Z (2020) Numerical investigation on the effects of the fracture network pattern on the heat extraction capacity for dual horizontal wells in enhanced geothermal systems. Geomech Geophys Geo-energ Georesour 6(1):1-17

Yu K, Zhou Y, Liu Y, Liu F, Hu L, Ao W, Xie H (2020) Nearroom-temperature thermoelectric materials and their application prospects in geothermal power generation. Geomech Geophys Geo-energ Geo-resour 6(1):12

Zhang Y, Zhao G-F (2020) A global review of deep geothermal energy exploration: from a view of rock mechanics and engineering. Geomech Geophys Geo-energ Geo-resour $6(1): 4$

Zhao J, Tang CA, Wang SJ (2020) Excavation-based enhanced geothermal system (EGS-E): introduction to a new concept. Geomech Geophys Geo-energ Geo-resour 6(1):6

Publisher's Note Springer Nature remains neutral with regard to jurisdictional claims in published maps and institutional affiliations. 